\title{
Pachydermodactyly - a report of two cases
}

\section{Zbigniew Żuber ${ }^{1}$, Grzegorz Dyduch², Andrzej Jaworek ${ }^{3}$, Dorota Turowska-Heydel ${ }^{1}$, Małgorzata Sobczyk ${ }^{1}$, Marta Banach-Górnicka ${ }^{1}$, Katarzyna Rusnak ${ }^{1}$, Wojciech Górecki ${ }^{4}$}

${ }^{1}$ Department of Older Children with subunits of Neurology, Rheumatology and Rehabilitation, St. Louis Regional Specialised Children's Hospital, Krakow, Poland

2Department of Pathological Anatomy, Jagiellonian University Medical College, Krakow, Poland

${ }^{3}$ Department of Dermatology, Jagiellonian University Medical College, Krakow, Poland

${ }^{4}$ Department of Children Surgery, Jagiellonian University Medical College, Krakow, Poland

\begin{abstract}
Pachydermodactyly (PDD) is a rare and benign form of digital soft tissues fibromatosis, which affects the skin of the fingers. The disorder is characterized by asymptomatic, symmetric, progressive soft tissue swelling of the proximal interphalangeal (PIP) joints of the fingers. The etiology of disease remains unknown. It is usually acquired, even though there are some publications that document family cases. It affects mainly adolescent men.

We report two boys with the bilateral swelling of the of the PIP joints of the fingers and skin and subcutaneous tissue thickening. Based on clinical manifestations, radiological study and histopathological examination, pachydermodactyly was diagnosed.

PDD is a rare and benign disorder, although it is important to consider other diseases, especially rheumatic conditions, in the differential diagnosis in order to avoid unnecessary additional tests and treatments.
\end{abstract}

Key words: pachydermodactyly, hands involvement, proximal interphalangeal joints.

\section{Introduction}

Pachydermodactyly (PDD) is a rare and benign form of digital soft tissues fibromatosis, which affects the skin of the fingers. The disorder is characterized by asymptomatic, symmetric, progressive soft tissue swelling of the proximal interphalangeal (PIP) joints of fingers II-IV and rarely $\mathrm{V}$. In some cases, the process of the expansion to the metacarpophalangeal joints (MCP) is observed, which is defined as transgrediens pachydermodactyly. There may be concomitance of skin lesions in the form of impetigo, erythema and desquamation, which can cause pain [1-6].

In PDD there are no lesions in the bones and periosteum in an X-ray examination. Also in synovium and joints in ultrasound examination no alterations are observed [6].The disorder was first described by Basex et al. in 1973 [7], and named by Verbov in 1975: pachy - thick, dermos - skin, dactylos - fingers [8].
So far in the literature only about 150 cases of PDD have been reported $[2-4,6,9]$. The etiology of the disease remains unknown, PDD is usually acquired, even though there are some publications that document family cases $[3,6,9,10]$. It affects mainly adolescent men [5]. It has been suggested that multiple mechanical traumas, such as the habit of interlacing and rubbing the fingers, could lead to skin thickness, what causes secondary, mechanical dermatosis based on compulsive behaviours [3-11]. The classic type of disease can even simulate polyarticular JIA with involvement of small joints of the hands [3, 8-10, 12].

The current classification was proposed by Bardazzi et al. [9] in 1998 and describes five types of pachydermodactyly: 1) classical, 2) localized - both associated with a local trauma, 3) transgrediens - reaching areas of metacarpophalangeal joints, 4) familial - which may be a transgrediens type, and 5) associated with tuberous sclerosis [9, 13]. 
Because the prevalence of PDD is sparse, we described two cases of this disorder, diagnosed among our patients.

\section{Case 1}

A 14.5-year-old boy was admitted to the hospital for the purpose of rheumatology diagnostics because of suspected connective tissue disease. Three years ago, after upper respiratory tract infection, the fingers thickening had been reported. He had no other symptomatic complaints, and impaired hand function was not observed, boy successfully attended a music school, played piano and stringed instruments. The mood and psychological development of the patient were normal.

A physical examination revealed: acne and many stretch marks on the back and thighs, on the hands: fusiform, bilateral, swelling of the lateral aspects of the PIP $(R>L)$ joints, significant skin and subcutaneous tissue thickening, skin above these lesions horny, rough and loose, slimming of the distal phalanges, skin above subtly thin, shiny, bilateral contractures and hyperkeratosis of the palmar surface of the hands. There were no vascular abnormalities of the skin of the hands (Figs. 1-3).

In the blood tests: complete blood count, percentage of the white blood cells, indicators of inflammation were in the reference range, rheumatoid factor (RF) was negative. Positive anti-nuclear antibodies (ANA) were reported (1: 640), extractable nuclear autoantibodies (ENA) were not found. In the ultrasound examination atypical image: bilateral, significant thickening of the periarticular soft tissues of the PIP II, III, IV finger joints (R > L), and a normal amount of synovial fluid. There were no signs of synovial hypertrophy or increased flow in color and power Doppler.

The X-ray image of bony structures and joint spaces was normal. It showed only spindle-shaped, symmetric soft tissues thickening around the PIP and DIP finger joints.

Similarly, in the magnetic resonance imaging (MRI) of the hands, there was observed bilateral subcutaneous tissue thickening around the PIP joints, after contrast administration - gain in signal intensity in this area, thickening and signal gain of DIP synovial membrane, with visible exudate and slight thickening of the digital flexor tendon sheath. In the capillaroscopy Raynaud's phenomenon was diagnosed (R-loops, atypical capillaries).

In the family history, the boy's father has a very similar morphology of the fingers - slimming of the distal phalanges, skin subtly thin, shiny, bilateral contractures and hyperkeratosis of the palmar surface of the hands, without periarticular (PIP) skin thickening. Due to the concomitance of different types of skin le-

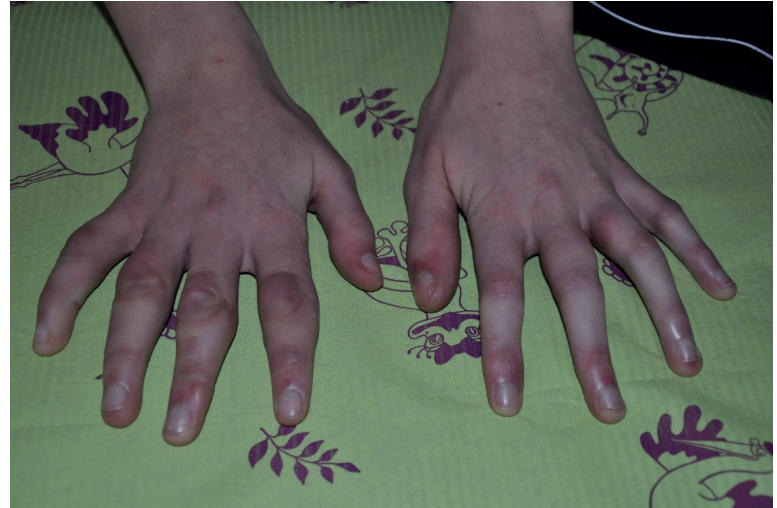

Fig. 1. In terms of hands: widening the outlines of the PIP joints of both hands $(P>L)$, a significant thickening of the skin and subcutaneous tissue, the skin above described changes hardened, rough, looses liding in small palpablenodules. Slim in terms of the distalphalanges, the skin taut, shiny, both sides contracture palmar hyperkeratosis of the palms of both hands.

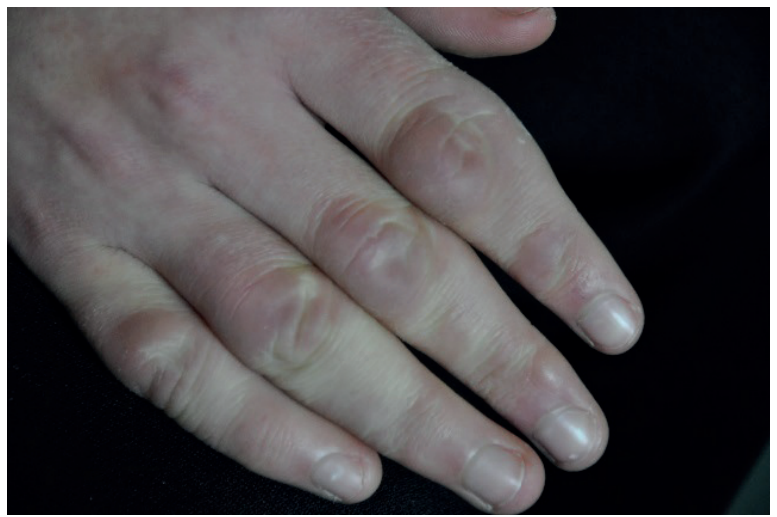

Fig. 2. Increased volume and thickening of proximal skin on the fingers.

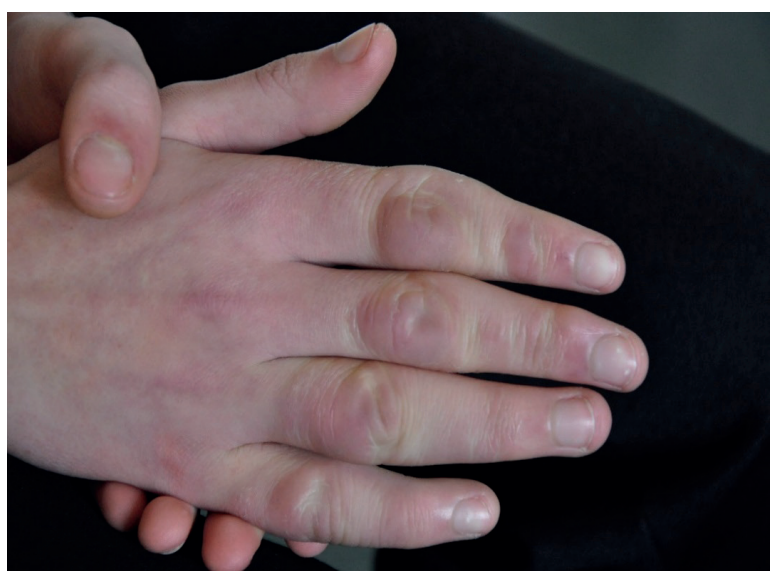

Fig. 3. Increased volume and thickening of proximal skin on the fingers. 


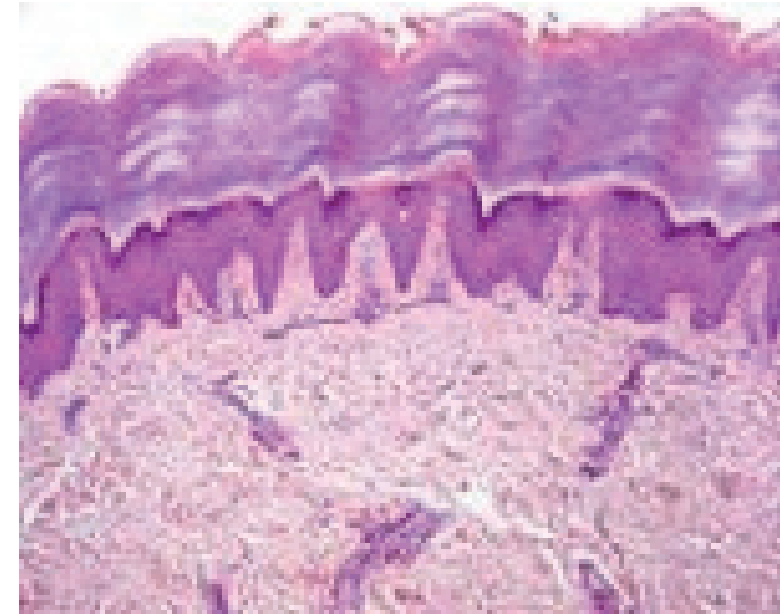

Fig. 4. (PIP joint region) Epidermis with features of hyperkeratosis and hypergranulosis, in the dermisslight infiltration of lymphocytes around blood vessels.

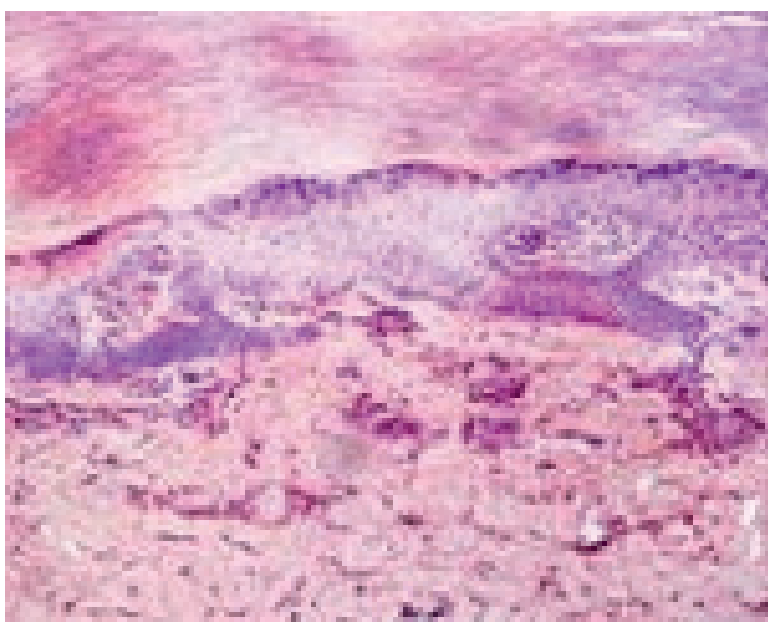

sions - around the PIP finger joints there was very dry and rough skin, with soft tissue thickening, while skin of distal phalanges was taut and shiny - the suspicion of sclerodactyly was suggested. In the dermatological consultation, two types of lesions were reported: hypertrophy of the periarticular (PIP) soft tissues of the right hand finger joints, in the distal phalanx region, paronychial induration, glittering, atrophy - observation suggested scleroderma. Histopathology was recommended. A skin lesions biopsy was performed and histopathological analysis revealed: around the PIP joint slight epidermal hyperplasia, hyperkeratosis of the skin, discrete proliferation of fibroblasts in the dermis, small deposits of mucin and sparse infiltrations of lymphocytes around the blood vessels (Figs. 4, 5) under the epidermis - proliferating capillaries and features of hyaline degeneration (Fig. 6).

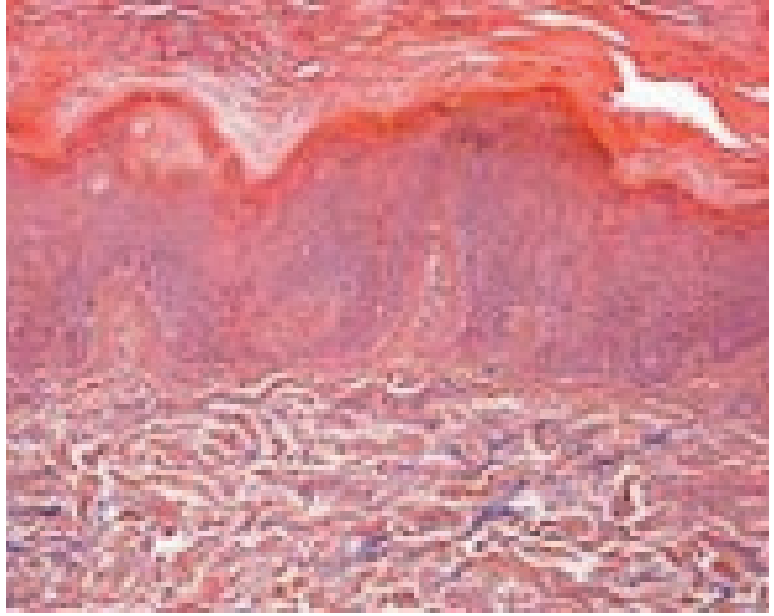

Fig. 5. (PIP joint region) Alcian blue stain to detect mucin (glycosaminoglycans) - small deposits of mucin in the dermis.

Fig. 6. (Paronychium) Epidermis with features of hyperkeratosis and focal hypergranulosis, under epidermis proliferation of capillaries and features of hyaline degeneration.

Based on clinical manifestation (presence of symptoms suggesting sclerodactyly), outcome of the disease, diagnostic tests (results of capillaroscopy and MRI examination, positive anti-nuclear antibodies titer), undifferentiated connective tissue disease (UCTD) with concomitance of symptoms suggesting pachydermodactyly was diagnosed [1, 3, 7, 14-17].

\section{Case 2}

A 15-year-old boy was admitted to the hospital for the purpose of rheumatology diagnostics because of suspected juvenile idiopathic arthritis (JIA). For 4 years bilateral thickening of the boy's fingers was observed. Symptoms had been preceded by a rash on the palmar surface of the hands. Currently, he has no other sympto- 
matic complaints. Because of the suspicion of arthritis of the fingers, the boy was released from physical education. Limited physical effort, especially in the hands, was recommended. He was treated with non steroidal anti-inflammatory drugs (NSAIDs).

A physical examination revealed: bilateral swelling of the PIP finger joints (II-V), significant skin and subcutaneous tissue thickening. There were no other $a b-$ normalities. In the blood tests: complete blood count, percentage of the white blood cells, indicators of inflammation (CRP, ESR), liver function tests and renal function parameters were in the reference range. Positive antinuclear antibodies (ANA) were reported (1:320). The ultrasound examination revealed: bilateral thickening of the soft tissues of the PIP I-V finger joints (articular fat pads), without features of arthritis, also the tendon sheaths without exudate.

In an X-ray image slight periarticular radiocarpal, intercarpal, carpometacarpal, MCP, bilateral PIP and DIP finger joints $(I-V)$ bone atrophy was reported. There was observed spindle-shaped, symmetric soft tissue thickening around the PIP and DIP finger joints. In order to allow histopathological analysis, a skin lesions biopsy was performed.

On the basis of clinical manifestation, imaging examination and histopathology (hyperkeratosis, hypergranulosis in epidermis, slightly thickened collagen fibers in the dermis) pachydermodactyly was diagnosed [1, 3, 7, 9].

\section{Discussion}

The diagnosis of pachydermodactyly is usually based on: characteristic clinical symptoms, correct results of laboratory tests, typical lesions in the imaging studies of hands, histopathological abnormalities and the exclusion of other potential causes of ailments.

Histological examination of a biopsy specimen shows mostly non - specific changes: epidermal hyperkeratosis with hypergranulosis, sometimes with thickening of the collagen fibers and disturbance of their linear system, slight proliferation of vascular superficial plexus with or without minimal inflammatory infiltration and occasionally small deposits of connective-tissue mucin [3].

The differential diagnoses of PDD include: polyarticular juvenile idiopathic arthritis (JIA) involving the PIP joints, rheumatoid arthritis (RA), subacute cutaneous lupus erythematosus (LE), fasciitis, limited scleroderma, diffuse systemic sclerosis (dSSc), obsessive "chewing pads", collagenous plaques of the hands, juvenile digital fibromatosis, progressive nodular fibrosis of the skin, foreign-body granuloma, thyroid disease, pachydermoperiostosis (Touraine-Solente-Golé syndrome), acromegaly, connective tissue nevi, fibrosing inflammatory conditions, Garrod's pads in violinists, acropachydermo- dactyly in psoriasis, paraneoplastic acropachydermodactyly, rheumatoid nodule and sarcomas $[4,5,9,10]$.

Treatment of pachydermodactyly consists of rehabilitation, which allows one to stop the progression or to reduce pathological changes. The beneficial effect of subcutaneous injections of glucocorticoids has been reported [18]. Because of the important role of mechanical trauma, stopping any compulsive mechanical skin irritation is recommended. Psychological support and, in some cases, psychiatric therapy are necessary [5, 11, 18]. Differential diagnosis of pachydermodactyly should consider atypical form of the disease, including one-sided occurrence and the possibility of the overlap syndrome with other connective tissue diseases [14-17].

Based on the complete clinical picture, outcome of the disease, and diagnostic test of the patient, in the case of the second boy pachydermodactyly was diagnosed. At this stage of development of the symptoms in the second patient, JA and other connective tissue diseases and syndromes that can give a picture similar to pachydermodactyly have been excluded $[2,7,12]$.

In the case of the first boy undifferentiated connective tissue disease (UCTD) with concomitance of symptoms of pachydermodactyly was diagnosed. At this stage of development of the symptoms and on the basis of results of diagnostic tests it is difficult to determine whether we are dealing with overlap syndrome or the development of symptoms of type PDD in the early stage of UCTD [14-17]. Typical histopathological hallmarks confirmed in both cases the PDD diagnosis. Rheumatologic observation is necessary because of the uncertainty as to the further development of disease symptoms, especially that the prognosis for the future depends on the development of UCTD symptoms [17].

In the second case, the low titer of anti-nuclear antibodies seems to be not associated with a systemic disease. However, it requires a periodic clinical follow-up. A low titer of ANA may also be detected in healthy patients or those with viral and bacterial infection $[19,20]$.

Other symptoms, reported in X-ray pictures, which require systematic diagnostic tests and further rheumatologic observation, are slight periarticular bone atrophies (probably caused by a long period of restricted hand mobility).

\section{Summary}

In a case of suspicion of PDD, it seems to be most important to exclude other potential causes of the presenting complaints, especially as the differential diagnosis includes many serious and intensively treated diseases. In the first place it requires differentiation with JIA with involvement of the small joints of the hands $[1,3,7,12,13]$. 
Correct and early diagnosis of PDD will allow many patients affected by this disease to avoid unnecessary invasive diagnostic procedures and anti-inflammatory or immunosuppressive treatment [11].

The authors declare no conflict of interest.

\section{References}

1. Hunt R, Mandal R, Walters R. Pachydermodactyly. Dermatol Online J 2010; 16: 5.

2. Chen CK, Shyur SD, Chu SH. Pachydermodactyly: Three new cases in Taiwan. J Microbiol Immunol Infect 2015; 48: 340-344.

3. Requena CB, Miot HA, Marques MEA. Case for diagnosis. Pachydermodactyly. An Bras Dermatol 2014; 89: 359-360.

4. Sandobal C, Kuznietz A, Varizat A. Pachydermodactyly: four additional cases. Clin Rheumatol 2007; 26: 962-964.

5. Beltraminelli H, Itin P. Pachydermodactyly - just a sign of emotional distress. Eur J Dermatol 2009; 19: 5-13.

6. Rachowska R, Filipowska-Grońska A, Weryńska-Kalemba M. Pachydermodactyly - a case report. Post Dermatol Alergol 2010; 27: 500-503.

7. Basex A, Dupre A, Teillard J. Pachydermie digitale des premieres phalanges par hyperplasie conjonctive dermique et aplasie hypodermique. Bull Soc Fr Dermatol Syphiligr 1973; 80: 455-456.

8. Verbov J. Letter: Pachydermodactyly: a variant of the true knuckle pad. Arch Dermatol 1975; 111: 524.

9. Bardazzi F, Neri I, Raone B. Pachydermodactyly: seven new cases. Ann Dermatol Venereol 1998; 125: 247-250.

10. Birman MV, Rossenwasser MP. Proximal interphalangeal joint fracture dislocations in professional baseball players. Hand Clin 2012; 28: 417-420.

11. Sinha NK, Ling SP, Nema SK, Pai DR. Pachydermodactyly does not need rheumatologic work-up. J Postgrad Med 2013; 59: 335-336.

12. Mesa-Del-Castillo Bermejo P, Martínez Menchón T, de la Peña Moral J. Classic pachydermodactyly. A simulator of juvenile idiopathic arthritis. An Pediatr (Barc) 2016; pii: S16954033(16)00028-X.

13. Nicolay JP, Faulhaber J, Kutzner H. A new subtype of pachydermodactyly: unilateral pachydermodactyly transgrediens. Clin Exp Dermatol 2015; 40: 633-635.

14. Laczik R, Soltesz P, Szodoray P. Impaired endothelial function in patients with undifferentiated connective tissue disease: a follow-up study Rheumatology (Oxford) 2014; 53: 2035 2043.

15. Pepmueller PH. Undifferentiated Connective Tissue Disease, Mixed Connective Tissue Disease, and Overlap Syndromes in Rheumatology. Mo Med 2016; 113: 136-140.

16. Nakken B, Bodolay E, Szodoray P. Cytokine Milieu in Undifferentiated Connective Tissue Disease: a Comprehensive Review. Clin Rev Allergy Immunol 2015; 49: 152-162.

17. Valentini G. Undifferentiated Connective Tissue Disease at risk for systemic sclerosis (SSc) (so far referred to as very early/early SSc or pre-SSc). Autoimmun Rev 2015; 14: 210-213.
18. Plana A, Bassas Vila J, Toro Montecinos M. Pachydermodactyly successfully treated with triamcinolone injections. Actas Dermosifiliogr 2014; 105: 319-321.

19. Puszczewicz M. Przeciwciała przeciwjądrowe - cóż z nimi począć? Reumatologia 2013; 51: 172-178.

20. Almagro MR, Rodríguez-Gutiérrez JF, Martín-Martínez MA. Association between antinuclear antibodies titers and connective tissue diseases in a Rheumatology Department. Reumatol Clin 2016 May. pii: S1699-258X(16)30015-8. 